\title{
Analyst
}

\section{Selective vancomycin detection using optical fibre long period gratings functionalised with molecularly imprinted polymer nanoparticles $\uparrow$}

\begin{abstract}
Sergiy Korposh, ${ }^{* a}$ Iva Chianella, ${ }^{\mathrm{b}}$ Antonio Guerreiro, ${ }^{\mathrm{b}}$ Sarah Caygill, ${ }^{\mathrm{b}}$ Sergey Piletsky, ${ }^{\mathrm{b}}$ Stephen W. James a and Ralph P. Tatam ${ }^{a}$

An optical fibre long period grating (LPG) sensor modified with molecularly imprinted polymer nanoparticles (nanoMIPs) for the specific detection of antibiotics is presented. The operation of the sensor is based on the measurement of changes in refractive index induced by the interaction of nanoMIPs deposited onto the cladding of the LPG with free vancomycin (VA). The binding of nanoMIPs to vancomycin was characterised by a binding constant of $4.3 \pm 0.1 \times 10^{-8} \mathrm{M}$. The lowest concentration of analyte measured by the fibre sensor was $10 \mathrm{nM}$. In addition, the sensor exhibited selectivity, as much smaller responses were obtained for high concentrations $(\sim 700 \mu \mathrm{M})$ of other commonly prescribed antibiotics such as amoxicillin, bleomycin and gentamicin. In addition, the response of the sensor was characterised in a complex matrix, porcine plasma, spiked with $10 \mu \mathrm{M}$ of VA.
\end{abstract}

Received 14th November 2013 Accepted 24th February 2014

DOI: $10.1039 / c 3 a n 02126 b$

www.rsc.org/analyst (i.e. chemical molecules or biological species that needs to be detected - analytes) in a polymeric matrix, achieved by its incorporation during the polymerization step. Molecular imprinting is achieved in three steps, the first being the complex formation of the template with selected functional monomers, followed by a polymerization step for creation of a matrix that fixes the monomers in the right position to interact with the analyte and with the final step being the removal of the template to leave behind cavities specific for the template, scheme 1.

Although MIPs have already being identified as stable and low cost mimics of receptors or enzymes for sensing applications, ${ }^{4-7}$ they are also perceived to have several shortcomings. Among these are the heterogeneous distributions of binding sites, which can produce high levels of non-specific binding and have complex protocols for integration with sensors, which can affect the reproducibility and hence increase the variability between measurements. Some of these issues can be resolved by the use of MIP nanoparticles (nanoMIPs) in standard protocols developed for the immobilisation of antibodies. The particles used here were produced by a solid-phase synthesis approach using a computer-controlled reactor. Such MIP nanoparticles have been shown to have pseudo-monoclonal binding properties, uniform binding sites and high affinity for the target used as the template. ${ }^{8,9}$ Recently, such MIP nanoparticles were used for the detection of vancomycin in a novel assay based on direct replacement of antibodies. ${ }^{10}$ The assay was capable of measuring vancomycin in buffer and in blood plasma within the range of $0.001-70 \mathrm{nM}$ with a detection limit of $0.0025 \mathrm{nM}$ (2.5 pM).
${ }^{a}$ Department of Engineering Photonics, School of Engineering, Cranfield University, Cranfield, Bedfordshire, MK43 OAL, UK. E-mail: s.korposh@cranfield.ac.uk ${ }^{b}$ Cranfield Health, Cranfield University, Cranfield, Bedfordshire, MK43 OAL, UK

$\dagger$ Electronic supplementary information (ESI) available. See DOI: $10.1039 / \mathrm{c} 3 \mathrm{an} 02126 \mathrm{~b}$ 


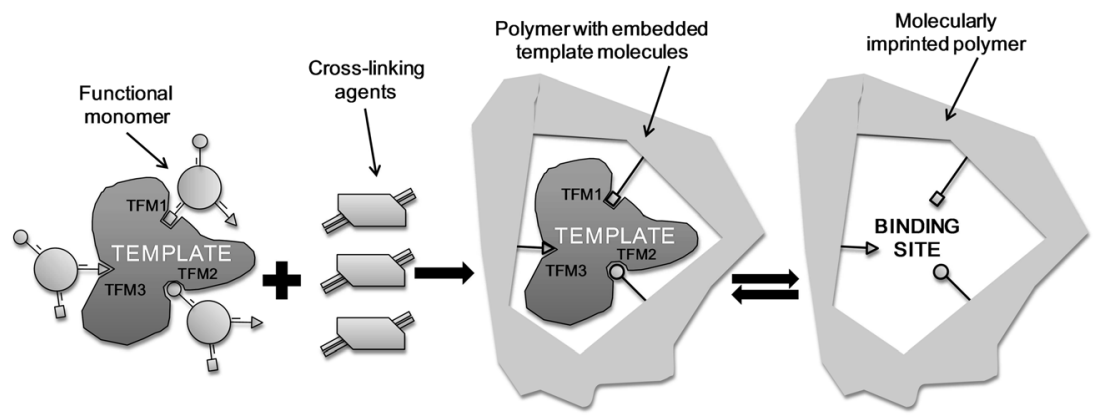

Scheme 1 Schematic illustration of the molecular imprinting procedure. ${ }^{3}$

In this work, nanoparticles produced following the protocol described in ref. 10 were deposited onto an optical fibre long period grating (LPG) with the aim of developing a low cost, highly sensitive and selective vancomycin sensor. Vancomycin is a glycopeptide antibiotic derived from Amycolatopsis orientalis. ${ }^{11}$ The mode of action of vancomycin consists of the inhibition of cell wall biosynthesis and alteration of the permeability of the bacterial cell membrane. Vancomycin has been used to treat various serious gram-positive infections. It is a powerful antibiotic, which in high doses can be toxic to the ears and kidneys, whilst at low doses can cause hypersensitivity reactions. Thus it is important to measure accurately the concentration of vancomycin in blood to control its administration to patients. The recommended therapeutic concentration range of vancomycin in plasma is $15-20 \mathrm{mg} \mathrm{L}^{-1} \cdot{ }^{12}$ Immunoenzymatic techniques (e.g. fluorescence polarisation immunoassay (FPIA)(TDx), enzyme multiplied immunoassay technique (EMIT), radioimmunoassay (RIA), turbidimetric assays) and chromatographic methods are the most commonly used in clinical environments for the detection of vancomycin in plasma. ${ }^{13}$ The former, although widely used because of their simplicity and speed, lacks precision, especially for the highest concentrations of the antibiotic. ${ }^{14,15}$ The latter can be very precise, but the techniques are time consuming and require expensive instruments and highly skilled operators. Therefore there is a need to develop reliable, fast, cheap and easy to use sensors for clinical measurements of vancomycin and antibiotics in general.

Regarding the sensor platform, a long period grating (LPG) is a periodic modulation of the refractive index (RI) of the core of an optical fibre that results in the coupling of light between core and cladding modes. Its combination with nanomaterials provides a prospect for the fabrication of high sensitivity sensors offering specific binding to targeted chemical species. ${ }^{16,17}$ Recently, we have demonstrated the use of optical fibre LPG sensors modified with different functional coatings for the detection of ammonia ${ }^{18}$ and aromatic carboxylic acids. ${ }^{19}$

The coupling between core and cladding modes occurs when the phase matching condition is satisfied:

$$
\lambda_{x}=\left(n_{\operatorname{core}}-n_{\operatorname{clad}(x)}\right) \Lambda
$$

where $\lambda_{x}$ represents the wavelength at which light is coupled to the $\mathrm{LP}_{0 x}$ cladding mode, $n_{\text {core }}$ is the effective refractive index of the mode propagating in the core of the fibre, $n_{\operatorname{clad}(x)}$ is the effective index of the $\mathrm{LP}_{0 x}$ cladding mode and $\Lambda$ is the period of the LPG.

The transmission spectrum of an optical fibre containing an LPG is characterised by a series of resonance bands centred at the wavelengths satisfying eqn (1). The dependences of the cladding modes effective refractive indices on the surrounding refractive index are manifested as changes in the central wavelengths of the resonance bands, which has been exploited as a refractive index sensor and non-specific concentration sensor, ${ }^{20}$ and by, coating with the cladding of the optical fibre in the section containing the LPG with a functional material, chemical sensors in both the liquid and gas phases.. ${ }^{17,18}$

The combination of optical fibre based sensing platforms with MIPs has already been attempted by several research groups. Previous reports have exploited surface plasmon resonance on metal coated optical fibres ${ }^{21,22}$ or have used a fluorescence signal, ${ }^{23}$ rather than LPGs. In all of these examples the MIP layer was produced in situ on the fibre, making large scale production of the optical sensors more challenging because of the need to control polymerization conditions to achieve high quality and reproducible MIP layers. In this paper, the MIP layer was deposited onto the optical fibre by simply immersing the optical fibre into a solution of soluble and easy-to-manipulate MIP nanoparticles. The nanoMIPs-modified optical fibre LPG was used to measure vancomycin in a concentration range from $10 \mathrm{nM}$ to $700 \mu \mathrm{M}$, equivalent to $14 \mu \mathrm{g} \mathrm{L}^{-1}$ and $1 \mathrm{~g} \mathrm{~L}^{-1}$, respectively. The lower concentration was chosen with the intention of assessing if this sensor was capable of measurements in dilutions of real samples, which would be helpful to reduce interferences when testing biological samples like plasma. The mid-range includes the recommended therapeutic concentration range, while the higher concentration was investigated to assess the concentrations at which the sensor response saturates. The selectivity of the sensor was tested against other commonly prescribed antibiotics such as gentamicin, amoxicillin and bleomycin. ${ }^{24}$ In addition, to check the sensor performance in a complex biological matrix, the response to vancomycin was also measured in porcine plasma spiked with the antibiotic.

\section{Experimental section}

\subsection{Materials}

$N$-Isopropylacrylamide (NIPAm), acrylic acid (AA), $N, N^{\prime}$-methylene-bis-acrylamide (BIS), N-tert-butylacrylamide (TBAm), 
ammonium persulfate (APS), tetramethylethylenediamine (TEMED), 3-aminopropyltrimethyloxysilane (APTMS), glutaraldehyde (GA), ethanolamine, phosphate buffered saline (PBS), methanol, ethanol, toluene and acetone were purchased from Sigma-Aldrich, UK. The antibiotics vancomycin (VA), amoxicillin, bleomycin sulphate salt, gentamicin sulphate salt (Scheme 2) were also from Sigma-Aldrich. $\mathrm{N}$-(3-Aminopropyl) methacrylamide hydrochloride (APMA) was from Polysciences Europe $\mathrm{GmbH}$, Germany. All the chemicals were of analytical grade, and used without further purification. Double-distilled ultrapure water (Millipore, UK) was used for the experiments. Phosphate buffered saline (PBS) was prepared as directed from PBS buffer tablets (Sigma-Aldrich, Gillingham, UK) and comprised phosphate buffer $(0.01 \mathrm{M})$, potassium chloride $(0.0027 \mathrm{M})$ and sodium chloride $(0.137 \mathrm{M}), \mathrm{pH} 7.4$, at $25{ }^{\circ} \mathrm{C}$. Where PBS, $\mathrm{pH} 7.2$, was used, the $\mathrm{pH}$ was adjusted by the addition of $\mathrm{HCl}$.

\subsection{Synthesis of MIP nanoparticles (nanoMIPs) imprinted with vancomycin}

The protocol for the immobilization of vancomycin on solid supports (glass beads) has been described elsewhere. ${ }^{8}$ An automated reactor, upgraded compared to that used previously, ${ }^{8}$ was utilized for the synthesis of the nanoparticles. During the entire synthesis the template remains attached to the glass beads. Once the high affinity nanoparticles, formed around the template, are collected with hot washing, the template still remains attached to the solid surface and the nanoparticles collected are already 'template free'. This is one of the major advantages of the solid support approach in comparison with other methods used for the synthesis of nanoparticles. Regarding the details of the automated synthesis, the reactor, manufactured by HEL Ltd., (Borehamwood, UK), was designed to allow both photochemically- and chemically-initiated polymerizations. All processes were controlled with a computer, with minimal intervention from the operator. For the preparation of nanoMIPs specific for vancomycin, the polymerization mixture, which was adapted from that published by Hoshino and colleagues ${ }^{25}$ consisted of NIPAm (39 mg), BIS ( $2 \mathrm{mg}$ ), TBAm (33 mg dissolved in $2 \mathrm{~mL}$ of ethanol), APMA (1.15 mg) and AA (2.23 g). The mixture was dissolved in double-distilled water $(100 \mathrm{~mL})$, sonicated for 10 minutes and degassed by bubbling with nitrogen for 30 minutes. For the automated synthesis of nanoMIPs, glass beads functionalized with vancomycin $(60 \mathrm{~g})$ were added to the reaction cylinder. Using a syringe pump, an aliquot of the polymerization mixture $(60 \mathrm{~mL})$ was added directly into the cylinder and then stirred briefly to homogenize the contents. Polymerization was initiated by the addition of a solution of APS $\left(60 \mathrm{mg} \mathrm{mL}^{-1}, 600 \mu \mathrm{L}\right)$ and TEMED $(18 \mu \mathrm{L})$ followed by stirring. The monomer mixture was left to polymerize at ambient temperature for 1.5 hours and a brief stirring (30 s at $600 \mathrm{rpm}$ ) was applied at the end of each $30 \mathrm{~min}$ period. At the end, the polymerization mixture was drained from the reaction vessel using a combination of suction and nitrogen purging. Subsequently, washings were performed at ambient temperature by adding water ( 2 aliquots of $50 \mathrm{~mL}$ ) to the cylinder. The contents were stirred and the washings, containing unreacted monomers and other low affinity materials, removed and discarded. Next, a further 3 aliquots of water (50 $\mathrm{mL}$ each) were individually added to the cylinder and the temperature was raised to $60{ }^{\circ} \mathrm{C}$. At this temperature pure

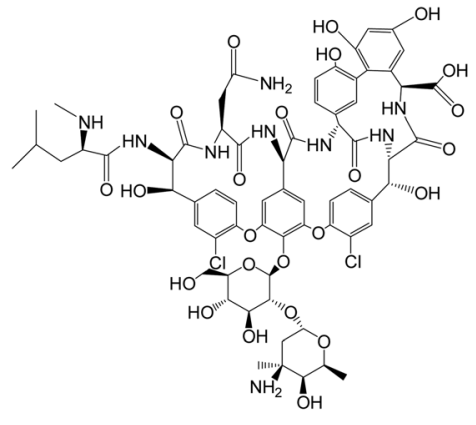

(a)

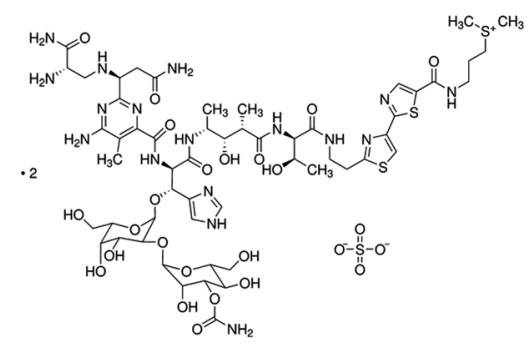

(c)

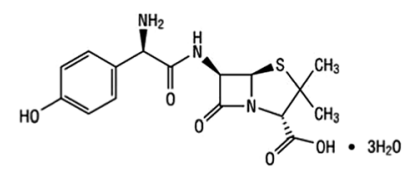

(b)

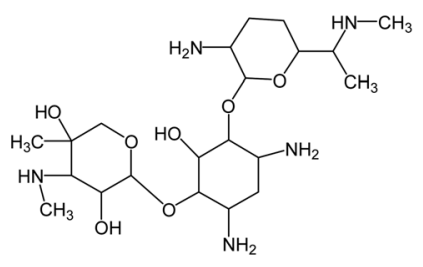

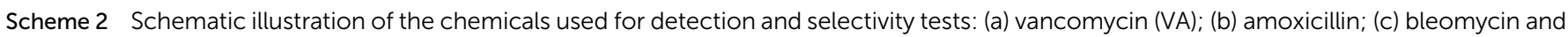
(d) gentamicin. 
fractions of nanoparticles were collected. The entire process took approximately 3.5 hours. The stages of the synthesis were pre-programmed into the WinISO ${ }^{\circledR}$ software such that, once started, the synthesis could be left to run without the necessity for user supervision. The solution of nanoMIPs was concentrated to a final volume of $100 \mathrm{~mL}$ by ultrafiltration on a Millipore Amicon Ultra centrifugal filter unit (30 kDa MWCO) and its concentration $\left(0.2 \mathrm{mg} \mathrm{mL}^{-1}\right)$ determined by weighing a freezedried aliquot. The size of the nanoparticles measured by dynamic light scattering (DLS) using a Zetasizer Nano (Nano-S) from Malvern Instruments Ltd (Malvern, UK) was around $280 \mathrm{~nm}$. Prior to DLS measurements, performed at $25^{\circ} \mathrm{C}$, the solution of nanoMIPs was subjected to sonication for 5 minutes.

\subsection{Sensor fabrication and functionalization}

An LPG with a length of $30 \mathrm{~mm}$ and a period of $111 \mu \mathrm{m}$ was fabricated in boron-germanium co-doped single mode optical fibre (Fibercore PS750) with a cut-off wavelength of $670 \mathrm{~nm}$, in a point-by-point fashion, side-illuminating the optical fibre by the output from a frequency-quadrupled Nd:YAG laser, operating at $266 \mathrm{~nm}$. The period of the LPG was chosen to ensure operation at the phase matching turning point, a condition known to offer the highest sensitivity. ${ }^{26}$ The fibre was fixed tightly in a Teflon cell as described previously. ${ }^{15-18}$ For transmission spectra (TS) measurements, one proximal end of the fibre was connected to a spectrometer (S200, Ocean Optics) and the distal end to a tungsten halogen lamp (HL-2000, Ocean Optics).

Prior to the deposition of the nanoMIPs, the region of the optical fibre containing the LPGs was rinsed with doubledistilled water and immersed into $1 \mathrm{wt} \%$ ethanolic $\mathrm{KOH}$ (ethanol-water $=3: 2, \mathrm{v} / \mathrm{v}$ ) for $20 \mathrm{~min}$, leading to formation of hydroxyl groups on the fibre surface. The fibre was then immersed into a $2 \% \mathrm{v} / \mathrm{v}$ solution of APTMS in dry toluene for different time intervals to find the optimal conditions, washed with acetone and subsequently incubated in a $1 \% \mathrm{v} / \mathrm{v}$ solution of GA in PBS ( $\mathrm{pH} 7.2$ ) for 1 hour. The LPG fibre was then rinsed with double-distilled water and immersed into a nanoMIPs solution $\left(0.2 \mathrm{mg} \mathrm{mL} \mathrm{mL}^{-1}\right)$ for $2 \mathrm{~h}$. After rinsing with doubledistilled water, the fibre was immersed for 30 minutes into an ethanolamine water solution $(0.1 \mathrm{M})$, to block the remaining free carbonyl groups of GA, and following a final wash with double-distilled water, the sensor was ready to be used. A schematic representation of the nanoMIPs immobilization protocol is reported on Scheme 3. The transmission spectrum (TS) of the LPG was measured after each step of the surface treatment.

\subsection{Sensitivity and selectivity tests}

After the functionalization, the sensor was exposed to variable concentrations of VA by immersion into $600 \mu \mathrm{L}$ solution for 45 min, as after this time there was no change in measured signal. This was followed by thorough rinsing with distilled water and drying by purging with dry nitrogen to remove any physically adsorbed compounds. The transmission spectrum (TS) of the LPG was measured after each exposure to analyte and interfering substances. To avoid issues with sensitivity of the LPG to the bulk refractive index, all TS measurements were conducted in air. In order to take into account the potential difference in the light source intensity between different measurements the TS were normalised by taking an automatic baseline for each measured TS using Origin 7.5 software. After each exposure, a solution of $\mathrm{NaOH}(0.1 \mathrm{M})$ was used to regenerate the sensor by removing the VA adsorbed to the nanoMIPs. The sensor selectivity was tested by exposure to other common antibiotics (amoxicillin, gentamicin and bleomycin) with a concentration of 600-700 $\mu \mathrm{M}$. The nanoMIPs-modified LPG was also

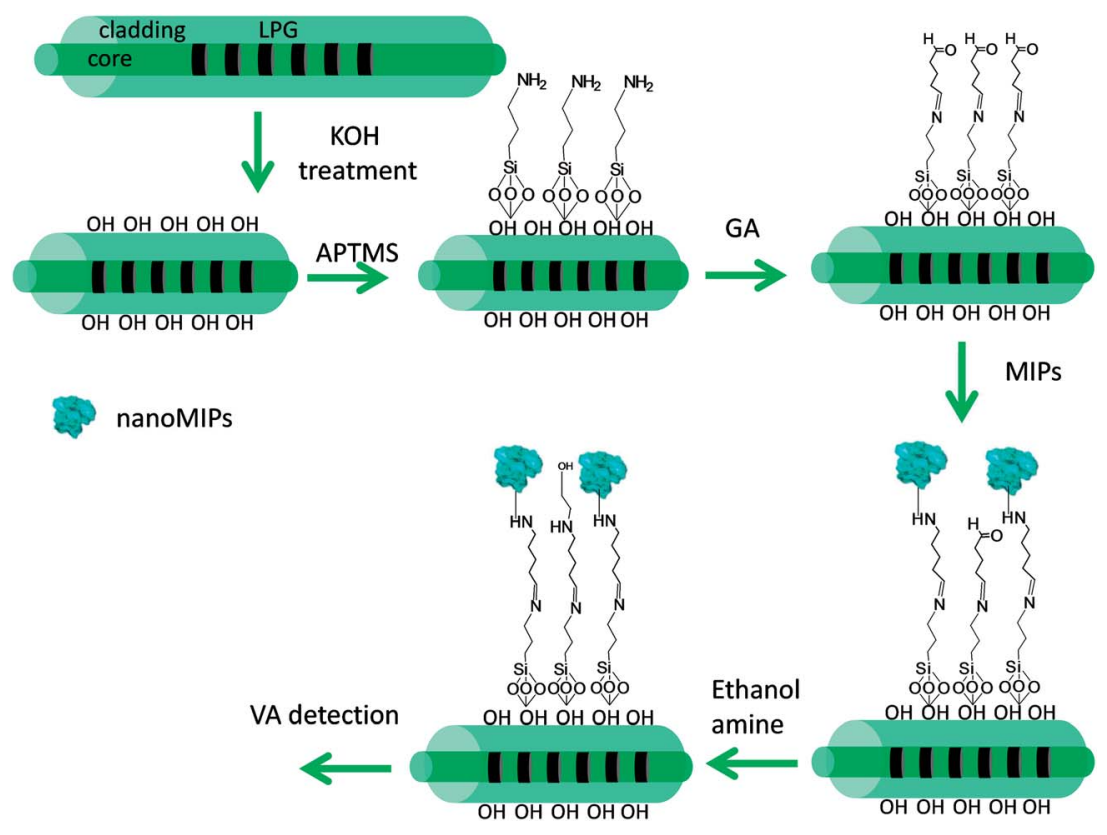

Scheme 3 Schematic illustration of the protocol used to immobilize nanoMIPs. 
immersed into porcine plasma spiked with $10 \mu \mathrm{M}$ of vancomycin to check the sensor performance in a complex biological matrix.

It should be noted that the effect of the non-imprinted nanoparticles was thoroughly studied in the previous work with ELISA assay ${ }^{10}$ where the imprinting effect of the nanoMIPs was clearly demonstrated and was not included in this current study.

\section{Results and discussion}

\subsection{NanoMIPs deposition}

The first step to immobilize covalently nanoMIPs onto the optical fibre involved the deposition of a thin silane film onto the hydroxyl groups on the fibre surface formed by the $\mathrm{KOH}$ ethanolic solution. To do this the fibre was immersed into $2 \% \mathrm{v} /$ $\mathrm{v}$ solution of APTMS in dry toluene. The silane also provides the surface with the primary amino groups necessary for the subsequent immobilization steps. Fig. 1 shows the TS of the LPG with grating period of $111 \mu \mathrm{m}$, measured in air before and after 1 hour deposition of the APTMS. It should be noted that, in order to take into account the potential difference in the intensity of the light source between different measurements (Fig. S1 $\dagger$ ), the TS were normalised by taking an automatic baseline for each measured TS using Origin 7.5 software. The blue shift of the linearly polarised (LP) mode $\mathrm{LP}_{018}$ resonance, along with the appearance of the dual resonance for the $\mathrm{LP}_{019}$ mode resonance, is characteristic of the response of the LPG to the deposition of a thin film onto the cladding of the fibre.

Following the deposition of the silane, the fibre was immersed into a PBS solution of GA, Fig. 2a and b. Similarly to that observed following the deposition of the APTMS film, a further blue shift of the $\mathrm{LP}_{018}$ resonance, and further development of the $\mathrm{LP}_{019}$ dual resonance bands were observed, demonstrating a successful deposition of GA via attachment of the carbonyl groups of GA to the amino groups of the silane. When measured in solution, the $\mathrm{LP}_{019}$ resonance band exhibited a significant wavelength shift during the first 15 min from the start of the GA deposition, with the response saturating at around $30 \mathrm{~min}$, as shown in Fig. 2a. Fig. 2b shows the TS measured in air before and after GA deposition, with the

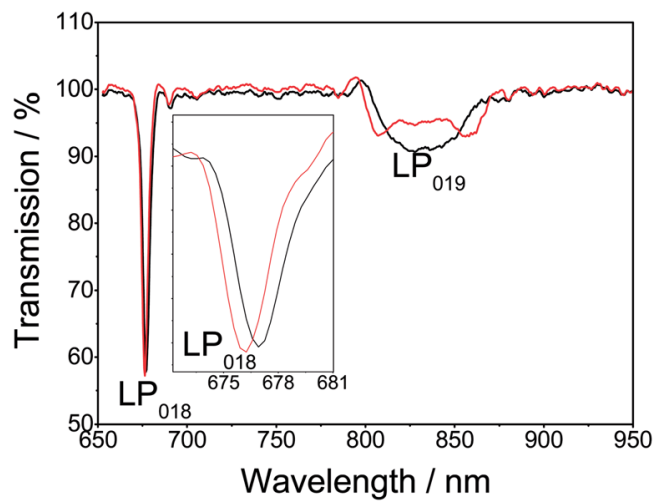

Fig. 1 TS of the LPG measured in air: black line, before APTMS deposition, and red line, after APTMS deposition.

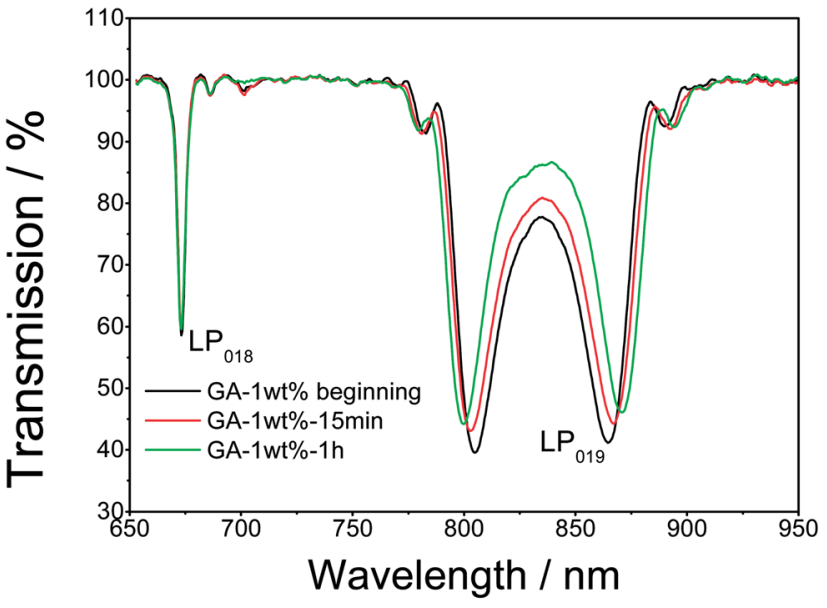

(a)

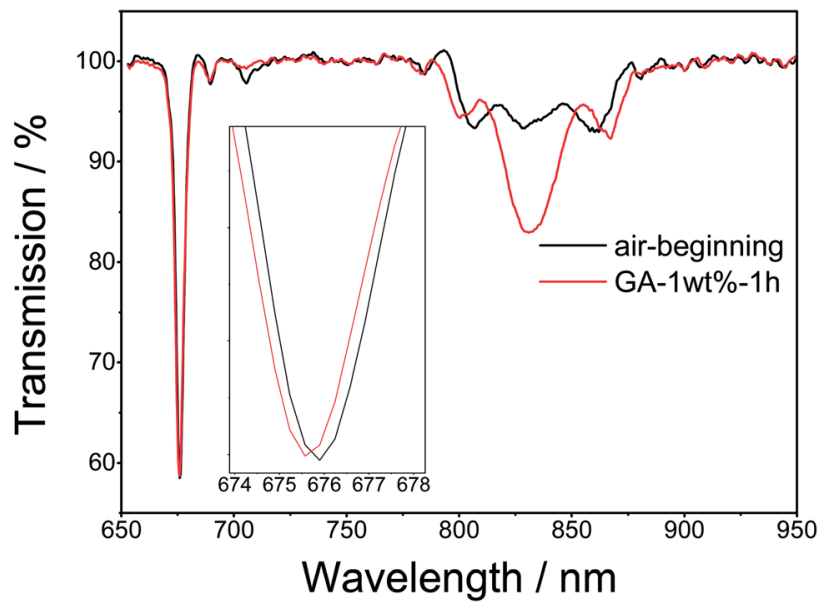

(b)

Fig. 2 (a) Evolution of the TS of the APTMS-coated optical fibre LPG during the deposition of $1 \mathrm{wt} \% \mathrm{GA}$, measured in solution at different interval times: black line, before GA deposition; red line, $15 \mathrm{~min}$; and green line, $1 \mathrm{~h}$ after GA deposition; (b) TS measured in air: black line, before GA deposition, and red line, after GA deposition.

changes in the spectra indicating an increase of the optical thickness of the film.

After GA deposition, the LPG was immersed into a water solution of nanoMIPs $\left(0.2 \mathrm{mg} \mathrm{mL}^{-1}\right)$ for 2 hours, Fig. S2. $\dagger$ The amino groups contained in the nanoMIPs (due to the monomer APMA) create a bond with the carbonyl groups of GA. The minor change in the $\mathrm{LP}_{018}$ resonance band apparent after the immobilization of nanoMIPs can be explained by successful attachment of the nanoparticles to the GA surface. The final step of the MIP sensor fabrication was the blocking by ethanolamine (0.1 M) of any available carbonyl groups remaining on GA, Fig. S3. $\dagger$ A small change in the TS was observed, revealing the adsorption of the ethanolamine.

\subsection{Vancomycin detection}

After the immobilization of the nanoMIPs and blocking with ethanolamine, the LPG was exposed to increasing 
concentrations of VA solution. Each concentration was incubated for 45 minutes (Fig. 3a) as, after this time, the single was saturated, Fig. S4. $\uparrow$ It should be noted, however, that these data cannot be directly used to assess amount that was bound, as the refractive index of the solution plays some role in the sensor response. The LPG was washed with $0.1 \mathrm{M} \mathrm{NaOH}$ after each exposure (Fig. S5†). As the concentration of VA increased, the change in the TS becomes more pronounced, indicating an increase in the amount of VA bound to the nanoMIPs. This behaviour of the TS of the coated LPG fibre is typical of that observed when the RI of the sensitive layer increases. ${ }^{15-18}$ The resonance band at $c a$. $670 \mathrm{~nm}$, which is coupled to the linearly polarized 18th cladding mode ( $\left.\mathrm{LP}_{018}\right)$, exhibits blue shifts of $0.22,0.40,0.60$ and $0.88 \mathrm{~nm}$ for the $0.01,0.1,10$ and $700 \mu \mathrm{M}$ VA concentrations, respectively. The transmission intensity of the resonance band at $c a .833 \mathrm{~nm}$, corresponding to coupling to the $\mathrm{LP}_{019}$ modes, decreases for $2.85,4.93,11.96$ and $15.63 \%$ for $0.01,0.1,10$ and $700 \mu \mathrm{M}$ VA concentrations, respectively, Fig. 3a. The sensor response starts to saturate at a concentration of VA of $700 \mu \mathrm{M}$, indicating the specific nature of VA binding, Fig. 3b.

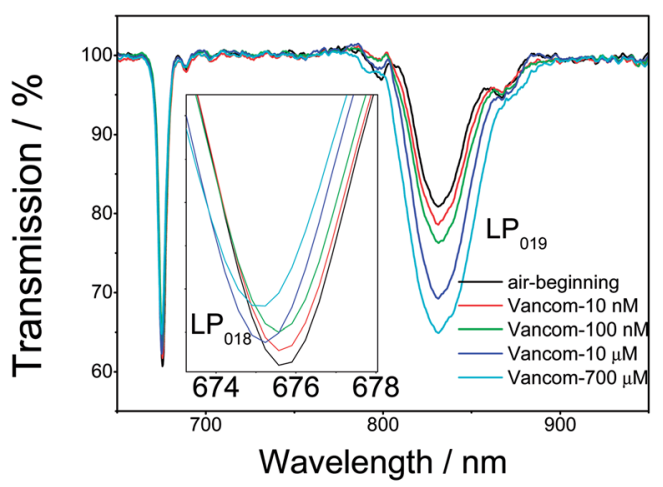

(a)

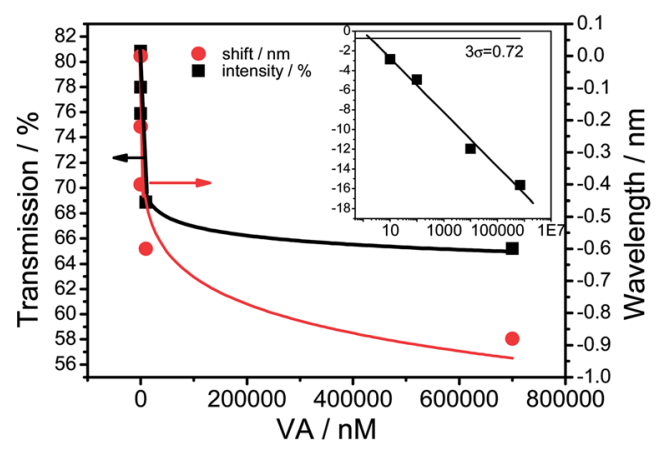

(b)

Fig. 3 (a) TS of the nanoMIP coated LPG measured in air after exposure to VA concentrations ranging from $10 \mathrm{nM}$ to $700 \mu \mathrm{M}$. (b) Dependence of the transmission at the centre of the resonance band corresponding to coupling to the $\mathrm{LP}_{019}$ mode (square) and wavelength shift of the resonance band corresponding to coupling to the $L P_{018}$ mode (circle) on the concentration of VA. The red and black lines are fits using the Langmuir adsorption isotherm. The inset shows the logarithmic dependence of transmission at the centre of the resonance band corresponding to coupling to the $\mathrm{LP}_{019}$ mode on VA concentration.
The main focus of the sensor characterisation was on concentrations lower than the physiological range (10 nM), demonstrating that this approach could be used to develop a sensor with sensitivity that would need dilutions of real samples before testing. This approach would have the effect of reducing interferences when testing biological samples like plasma.

When the nanoMIP-modified LPG was regenerated with $\mathrm{NaOH}(0.1 \mathrm{M})$ the signal returned to its original value, indicating total removal of VA from the nanoMIPs, Fig. S5. $\dagger$ The binding of VA to the nanoMIPs was fitted with a Langmuir model using the standard package available in Origin software (Fig. $3 \mathrm{~b}$ and $\mathrm{S} 6 \dagger$ ). The binding constant of VA to the nanoMIPs film, calculated using a Scatchard-Rosenthal plot in accordance with the eqn (2) (Fig. S5 $\dagger$ ), was $4.3 \pm 0.01 \times 10^{-8} \mathrm{M}^{27}$

$$
\frac{B_{\max }}{B}=K_{\mathrm{d}} \frac{1}{c}+1,
$$

where $B_{\max }$ is the maximum amount of VA bound (sensor response at highest concentration), $B$ is the amount of VA bound at a given concentration (sensor response at a given concentration), $K_{\mathrm{d}}$ is the binding constant and $c$ is the concentration of VA.

The standard deviation, $\sigma$, obtained from 5 exposures to blank sample (Fig. S6 $\dagger$ ) and $10 \mu \mathrm{M}$ of VA, was $\pm 0.24 \%$ and $\pm 0.12 \mathrm{~nm}$ for the transmission and wavelength measurements, respectively. For transmission measurements, the limit of detection of the sensor system was found to be $1.8 \mathrm{nM}$, determined by calculating the $3 \sigma$ value. $^{27}$

Fig. 4 shows the TS measured in air before and after exposure (for 45 minutes) to porcine plasma with and without VA $(10 \mu \mathrm{M})$. A small sensor response was observed for plasma without VA. The response increased when $10 \mu \mathrm{M}$ of VA was present in the plasma, Fig. 4. These results suggest that the sensor can reliably perform VA detection in a complex medium such as plasma. It should be noted, however, that response to $10 \mu \mathrm{M}$ of VA was larger when measured in plasma as compared to VA in PBS. The most plausible explanation for this is the effect of the complex medium on the sensor response; this phenomenon could be

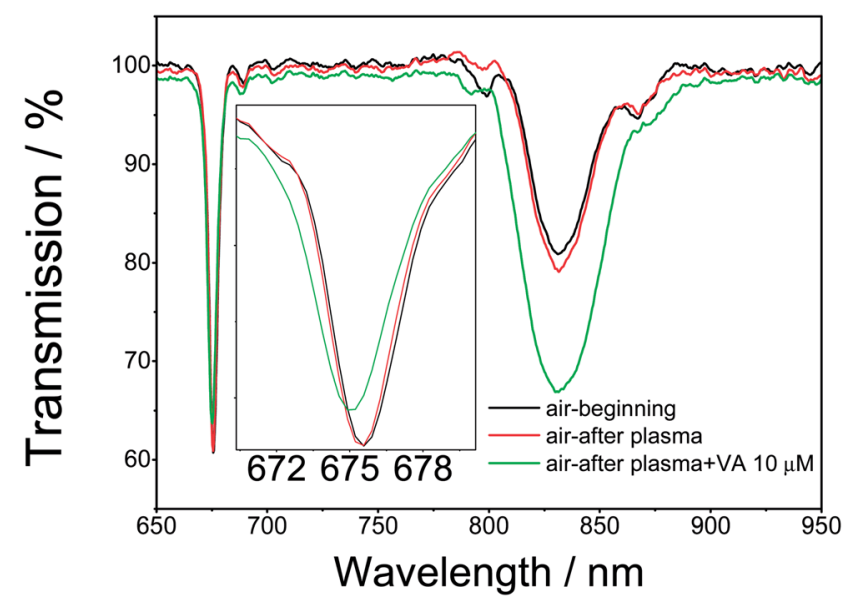

Fig. 4 TS of the nanoMIP coated LPG, measured in air before (black line) and after exposure to, porcine plasma (red line) and porcine plasma spiked with $10 \mu \mathrm{M}$ of VA (green line). 


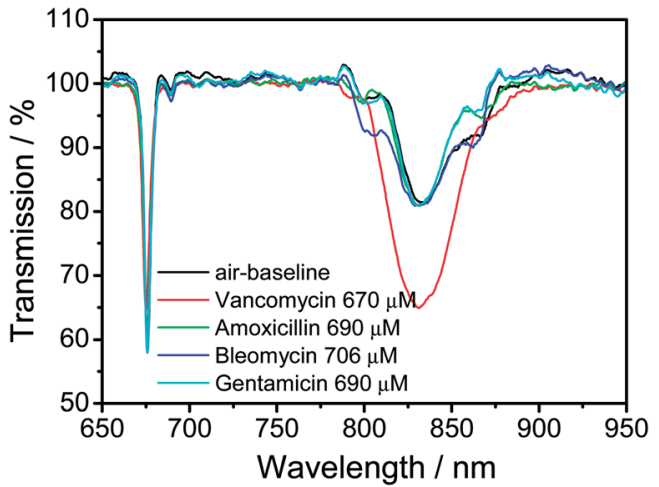

Fig. 5 TS of the nanoMIP-coated LPG, measured in air after exposure to, VA $(670 \mu \mathrm{M})$ (red line), amoxicillin $(690 \mu \mathrm{M})$ (green line); bleomycin $(706 \mu \mathrm{M})$ (blue line) and gentamicin $(690 \mu \mathrm{M})$ (magenta line).

solved either by further optimisation of the blocking of the particles or by performing an antibiotic calibration curve directly in plasma which should negate the effect of the complex medium. This will be studied in more detail in future work.

\subsection{Selectivity test}

When the nanoMIPs-modified LPG was exposed to solutions of amoxicillin $(610 \mu \mathrm{M})$, bleomycin $(700 \mu \mathrm{M})$ and gentamicin $(660 \mu \mathrm{M})$, each prepared in PBS, no measurable changes in the central wavelength of the resonance band corresponding to coupling to the $\mathrm{LP}_{018}$ mode, nor of the transmission at the centre of the resonance band corresponding to coupling to the $\mathrm{LP}_{019}$ mode were observed, revealing the high selectivity of the LPG sensor towards other commonly prescribed antibiotics, (Fig. 5).

\section{Conclusions}

In conclusion, molecularly imprinted polymer nanoparticles specific for VA were successfully deposited for the first time onto an optical fibre long period grating. The sensor demonstrated high sensitivity $\left(10 \mathrm{nM}\right.$ with $4.3 \pm 0.01 \times 10^{8} \mathrm{M}^{-1}$ binding constant) to VA and selectivity over other antibiotics such as amoxicillin, bleomycin and gentamicin. Successful detection of VA in a complex biological matrix, porcine plasma was also demonstrated. The LPG sensor based on molecular imprinted nanoparticles presented here is a promising tool for detection of antibiotics in biological fluids. In addition, the generic nature of both the LPG sensor and the nanoMIP prepared by solid-phase synthesis suggests that by immobilizing different nanoMIPs (produced against a different analyte), low cost and easy to use optical fibre sensors can be produced for applications in a variety of fields, from medical and environmental to food and industry.

\section{Acknowledgements}

The authors thank The Wellcome Trust (UK) for the financial support through the granting of a Translational Award and the EPRSC Platform Grant (EP/H02252X/1).

\section{References}

1 H. M. R. Goncalves, A. J. Duarte, F. Davis and S. P. J. Higson, Anal. Chim. Acta, 2012, 735, 90.

2 M. Consales, S. Campopiano, A. Cutolo, M. Penza, P. Aversa, G. Cassano, M. Giordano and A. Cusano, Meas. Sci. Technol., 2006, 17, 1220.

3 S.-W. Lee, S. Korposh, R. Selyanchyn and T. Kunitake, in Handbook of Molecular Imprinting: Advanced Sensor Applications, ed. S.-W. Lee and T. Kunitake, Pan Stanford Publishing Pte Ltd, 2012, ch. 1, pp. 1-64.

4 M. J. Whitcombe, I. Chianella, L. Larcombe, S. A. Piletsky, J. Noble, R. Porter and A. Horgan, Chem. Soc. Rev., 2011, 40, 1547.

5 C. Malitesta, E. Mazzotta, R. A. Picca, A. Poma, I. Chianella and S. A. Piletsky, Anal. Bioanal. Chem., 2011, 402, 1827.

6 G. Diaz-Diaz, D. Antuna-Jimenez, M. C. Blanco-Lopez, M. J. Lobo-Castanon, A. J. Miranda-Ordieres and P. TunonBlanco, TrAC, Trends Anal. Chem., 2012, 33, 68.

7 Y. Uludag, S. A. Piletsky, A. P. F. Turner and M. A. Cooper, FEBS J., 2007, 274, 5471.

8 A. Poma, A. Guerreiro, M. J. Whitcombe, E. V. Piletska, A. P. F. Turner and S. A. Piletsky, Adv. Funct. Mater., 2013, 23, 2821.

9 E. Moczko, A. Poma, A. Guerreiro, I. Perez de Vargas Sansalvador, S. Caygill, F. Canfarotta, M. J. Whitcombe and S. Piletsky, Nanoscale, 2013, 5, 3733.

10 I. Chianella, A. Guerreiro, E. Moczko, J. S. Caygill, E. V. Piletska, I. M. Perez De Vargas Sansalvador, M. J. Whitcombe and S. A. Piletsky, Anal. Chem., 2013, 85, 8462.

11 M. Rybak, B. Lomaestro, J. C. Rotschafer, R. Moellering, W. Craig, M. Billeter, J. R. Dalovisio and D. P. Levine, Am. J. Health-Syst. Pharm., 2009, 66, 82.

12 J. Truong, B. J. Levkovich and A. A. Padiglione, Intern. Med. J., 2012, 42, 23.

13 M. J. de Jesus Valle, F. Gonzalez Lopez and A. Sanchez Navarro, J. Pharm. Biomed. Anal., 2008, 48, 835.

14 K. A. Rodvold, S. M. Erdman and R. D. Pryka, in Therapeutic drug monitoring, ed. G. E. Schumacher, Appleton and Lange, Norwalk, CT, 1995, pp. 587-632.

15 S. A. Simons, A. R. Molinelli, K. Sobhani, P. M. Rainey and A. N. Hoofnaglea, Clin. Chem., 2009, 55, 578.

16 S. Korposh, S.-W. Lee, S. W. James and R. P. Tatam, Meas. Sci. Technol., 2011, 22, 075208 (10 p).

17 S. Korposh, S. W. James, S. W. Lee, S. Topliss, S. C. Cheung, W. J. Batty and R. P. Tatam, Opt. Express, 2010, 18, 13227.

18 T. Wang, S. Korposh, R. Wong, S. James, R. Tatam and S. W. Lee, Chem. Lett., 2012, 41, 1297.

19 S. Korposh, T. Wang, S. James, R. Tatam and S. W. Lee, Sens. Actuators, B, 2012, 173, 300.

20 R. Falciai, A. G. Mignani and A. Vannini, Sens. Actuators, B, 2001, 74, 74.

21 R. Verma and B. D. Gupta, Sens. Actuators, B, 2013, 177, 279.

22 N. Cennamo, G. D’Agostino, R. Galatus, L. Bibbò, M. Pesavento and L. Zeni, Sens. Actuators, B, 2013, 188, 221.

23 T. H. Nguyen, S. A. Hardwick, S. Tong and K. T. U. Grattan, IEEE Sens. J., 2012, 12, 255. 
24 K. Fujiwara, Y. Yoshizaki, M. Shin, T. Miyazaki, T. Saita and S. Nagata, Antimicrob. Agents Chemother., 2012, 56, 5883.

25 Y. Hoshino, T. Urakami, T. Kodama, H. Koide, N. Oku, Y. Okahata and K. J. Shea, Small, 2009, 5, 1562.
26 S. C. Cheung, S. M. Topliss, S. W. James and R. P. Tatam, J. Opt. Soc. Am. B, 2008, 25, 897.

27 A. Bossi, S. A. Piletsky, E. V. Piletska, P. G. Righetti and A. P. F. Turner, Anal. Chem., 2001, 73, 5281. 